\title{
Probing flavor changing neutral Higgs couplings at LHC
}

\author{
Wei-Shu Hou ${ }^{a}$, Guey-Lin Lin ${ }^{b}$, Chien-Yi Ma ${ }^{c}$, C.-P. Yuan ${ }^{d}$ \\ a Department of Physics, National Taiwan University, Taipei, Taiwan, R.O.C. \\ ${ }^{b}$ Institute of Physics, National Chiao Tung University, Hsinchu, Taiwan, R.O.C. \\ ${ }^{c}$ Department of Electrophysics, National Chiao-Tung University, Hsinchu, Taiwan, R.O.C. \\ d Department of Physics and Astronomy, Michigan State University, East Lansing, MI 48824, USA.
}

Received 24 June 1997

Editor: H. Georgi

\begin{abstract}
Uniquely heavy with mass at the weak scale, the top quark may have large flavor changing couplings to Higgs bosons that are as yet unexplored. We show that such couplings could be directly probed at the LHC through the parton subprocess $c(\bar{c}) g \rightarrow t(\bar{t}) A^{0}$, where the pseudoscalar $A^{0}$ subsequently decays into $t \bar{c}$ or $\bar{t} c$, giving rise to the intriguing final state of like sign top quark pairs. After demanding $\ell^{ \pm} \ell^{ \pm}$, missing energy and two $b$-jets, the major background turns out to be $q \bar{q}^{\prime} \rightarrow W l \bar{l}$, which can be partially suppressed by jet counting. The signal can then manifest itself in the asymmetry of numbers of $\ell^{+} \ell^{+}$and $\ell^{-} \ell^{-}$events. To further improve the signal over background, efficient $t$ vs. $\bar{t}$ tagging methods should be developed. (C) 1997 Elsevier Science B.V.
\end{abstract}

PACS: 14.80.Dq; 14.80.Gt; 12.15.Cc; 13.90.+i

Despite spectacular agreement with experiment, the Standard Model (SM) offers limited insight into its own structure. In particular, it does not explain but simply parametrizes the hierarchical patterns seen in both the fermion masses and the CKM mixing matrix. Nor does it reveal any details of the Higgs sector which is responsible for the electroweak symmetry breaking. Before a theory to account for these issues can be established, it is essential to gather experimental hints by exploring the properties of the top quark and the Higgs boson(s). Since $m_{t}$ is of the same order as the electroweak symmetry breaking scale, flavor dynamics involving the top quark and the electroweak symmetry breaking mechanism might be closely related to each other. In this note we examine the case where the top quark possesses large flavor changing couplings to neutral Higgs bosons [1,2].
To focus our discussions, we adopt the scenario that electroweak symmetry breaking is driven by a scalar sector. Given stringent limits on flavor changing neutral current (FCNC) processes, the structure of this scalar sector beyond its simplest form in SM is very restricted. In multi-Higgs doublet models, it is customary to impose some discrete symmetries [3] to ensure the absence of flavor changing neutral Higgs couplings (FCNH) at the tree level. However, inspired by the quark mass and mixing hierarchy pattern,

$$
\begin{gathered}
m_{1} \ll m_{2} \ll m_{3}, \\
\left|V_{u b}\right|^{2} \ll\left|V_{c b}\right|^{2} \ll\left|V_{u s}\right|^{2} \ll 1 .
\end{gathered}
$$

Cheng and Sher [4] suggested that low energy FCNC can be naturally suppressed without invoking discrete symmetries. For example, in the general two-Higgs 
doublet model (2HDM), quark mass matrices consists of two parts, $\boldsymbol{m}=\boldsymbol{m}^{(1)}+\boldsymbol{m}^{(2)}$, where $\boldsymbol{m}^{(1)}$ and $\boldsymbol{m}^{(2)}$ are each induced by vacuum expectation values (v.e.v.): $v_{1}=\left\langle\Phi_{1}^{0}\right\rangle$ and $v_{2}=\left\langle\Phi_{2}^{0}\right\rangle$. To sustain (1), unless fine-tuned cancellations occur, the offdiagonal elements of $\boldsymbol{m}^{(1)}$ and $\boldsymbol{m}^{(2)}$, just like $\boldsymbol{m}$ itself, should trickle off as one moves off-diagonal. Hence, the FCNH coupling matrices $\boldsymbol{\xi}^{(k)}$, obtained from $\sqrt{2} \boldsymbol{m}^{(k)} / v_{k}$ by rotating to the mass eigenbasis, cannot be arbitrary. Based upon this observation, Cheng and Sher proposed [4] the ansatz $\xi_{i j}^{(k)} \sim \sqrt{m_{i} m_{j}} / v_{k}$. Thus, FCNH couplings involving lower generation fermions are naturally suppressed, without pushing FCNH Higgs boson masses to way beyond the v.e.v. scale. However, since $\sqrt{2} m_{t} \cong v \equiv \sqrt{v_{1}^{2}+v_{2}^{2}}$, the flavor changing coupling $\xi_{t c}^{(k)}$ could be quite sizable, and could hence lead to interesting consequences such as $t \rightarrow c+S^{0}[1,2]$ or $S^{0} \rightarrow t \bar{c}, \bar{t} c$ [1], where $S^{0}$ is some neutral Higgs boson. Since top decay seems to proceed predominantly via $t \rightarrow b W^{+}$, we shall be interested in the case where neutral Higgs bosons are heavier than the top quark.

Before we go on to discuss how to probe $\xi_{t c}^{(k)}$, let us rotate $\Phi_{1}$ and $\Phi_{2}$ [5] such that $\left\langle\phi_{2}^{0}\right\rangle=0$ and $\left\langle\phi_{1}^{0}\right\rangle=$ $v / \sqrt{2}$. This eliminates $\xi^{(1)}$ and transforms $\xi^{(2)}$ into

$\xi_{i j}=f_{i j} \sqrt{m_{i} m_{j}} / v$,

where $f_{i j}$ 's are constants of order unity. In this new basis, the pseudoscalar $A^{0} \equiv \sqrt{2} \operatorname{Im} \phi_{2}^{0}$ and charged scalar $H^{ \pm} \equiv \phi_{2}^{ \pm}$are physical Higgs bosons. The CP even neutral scalars $\sqrt{2} \operatorname{Re} \phi_{1}^{0}$ and $\sqrt{2} \operatorname{Re} \phi_{2}^{0} \mathrm{mix}$ through the Higgs potential into the physical states $H^{0}$ and $h^{0}$. In the limit that the mixing angle $\sin \alpha \rightarrow 0$, $H^{0} \rightsquigarrow \sqrt{2} \operatorname{Re} \phi_{1}^{0}$ becomes the "standard" Higgs boson with diagonal couplings, while $h^{0} \rightsquigarrow \sqrt{2} \operatorname{Re} \phi_{2}^{0}$ has Yukawa couplings as in (2), but decouples from vector or $H^{+} H^{-}$boson pairs, just like $A^{0}$.

For $m_{A^{0}}<m_{H^{+}}+M_{W}$ and $m_{h^{0} / H^{0}}+M_{Z^{0}}$ (easily realized if all Higgs bosons have mass $\sim v$ ), and assuming that $\mathrm{CP}$ is a good approximate symmetry, the $A^{0}$ is unique in that it decays only into fermionic final states. Between the $t \bar{c}$ and $t \bar{t}$ thresholds,

$200 \mathrm{GeV}<m_{A^{0}}<2 m_{t} \simeq 350 \mathrm{GeV}$,

with the coupling of (2), $A^{0} \rightarrow t \bar{c}, \bar{t} c$ would dominate over the usual $b \bar{b}$ mode and become dominant [1]. We shall therefore concentrate on $A^{0}$ in this mass range as an FCNH probe.

The signatures of sizable $\xi_{t c}$ coupling at $\ell^{+} \ell^{-}$colliders have been discussed recently $[6,7]$. In particular, it was suggested [7] that at the $500 \mathrm{GeV}$ Next Linear Collider (NLC), the process $e^{+} e^{-} \rightarrow Z^{*} \rightarrow$ $h^{0} A^{0} \rightarrow t \bar{c} t \bar{c}$ or $\bar{t} c \bar{t}_{c}$ could lead to an intriguing final state with like sign top quark pairs, the traditional hallmark of neutral meson mixings (note that $T_{q}=t \bar{q}$ mesons do not even form). With both $m_{h^{0}}$ and $m_{A^{0}}$ in range of (3), and if $\sin \alpha \rightarrow 0$, the $t t \bar{c} \bar{c}$ ( $\bar{t} \bar{t} c c$ ) final state is most favorable since both $h^{0}$ and $A^{0}$ dominantly decays into $t \bar{c}(\bar{f} c)$. However, with an integrated luminosity of $50 \mathrm{fb}^{-1}$, one expects no more than a score of like sign dilepton events at NLC per year, which improves if $\sqrt{s}$ can be raised. For generic $\sin \alpha$ values where $h^{0} \rightarrow W W, Z Z$ decays are dominant, the like sign top final state is no longer favorable, but like sign dilepton events are still expected from the $W^{+} W^{-} t \bar{c}(\bar{t} c)$ final state. Note that the strength of $\xi_{t c}$ is only indirectly probed through the decays of $h^{0}$ and $A^{0}$ here. To probe $\xi_{t c}$ directly and with a larger event rate, we turn to hadronic colliders.

It is instructive to compare the event rates of $h^{0} A^{0}$ production at linear and hadronic colliders. At Tevatron, one expects no gain for $q \bar{q} \rightarrow h^{0} A^{0}$ since production cross section is similar, but the luminosity at a few $\mathrm{fb}^{-1}$ per year with the Main Injector is smaller than at the NLC. The situation is only slightly improved at the LHC. The cross section of $h^{0} A^{0}$ production is about $7 \mathrm{fb}$ for $m_{A^{0}}=m_{h^{0}}=250 \mathrm{GeV}$. With an integrated luminosity of $100 \mathrm{fb}^{-1}$, there are 700 pairs of $h^{0} A^{0}$ produced each year, which is not much better than $\sim 300 h^{0} A^{0}$ pairs [7] produced at an NLC with energy extended to $600 \mathrm{GeV}$ (assuming $50 \mathrm{fb}^{-1}$ ), but facing more backgrounds.

Hadronic colliders, however, offer the opportunity to involve the strong interaction in the production process, which can be used to directly probe $\xi_{t c}$. Surveying $q \bar{q}, q g$ and $g g$ processes, to have $\xi_{c}$ appearing in one of the interaction vertices, in general one requires $2 \rightarrow 3$ scattering, such as $q \bar{q} \rightarrow g^{*} \rightarrow t \bar{c}(\bar{t} c) A^{0}$. However, the cross sections turn out to be very small, and it would be advantageous if $2 \rightarrow 2$ scattering is possible. We find that the $c(\bar{c}) g \rightarrow t(\bar{l}) A^{0}$ process is rather promising in this regard as a direct probe to $\xi_{t c}$. Although the cross section at Tevatron remains small, the situation changes drastically at LHC. 


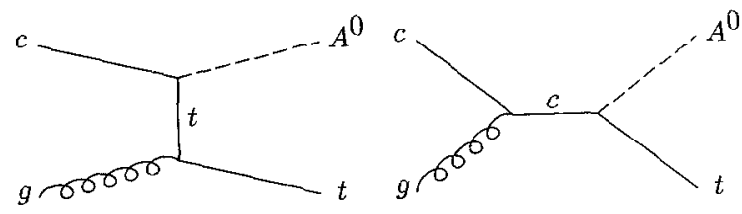

Fig. 1. Subprocess $c g \rightarrow t A^{0}$.

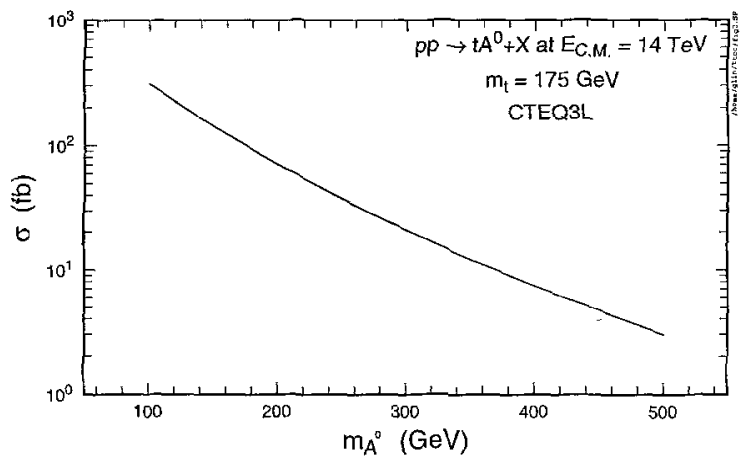

Fig. 2. Cross section for $p p \rightarrow t A^{0}+X$ at LHC via subprocess of Fig. 1.

At Tevatron cnergies, to producc an $A^{0}$ of $250 \mathrm{GeV}$ in association with a top quark, the colliding partons must carry large momentum fractions, hence both charm and gluon distribution functions are very suppressed, resulting in a very small $t A^{0}$ production cross section. From Fig. 1 and using CTEQ3L [8] parton distribution functions, the cross section at the Tevatron is only about $10^{-7} f^{2} \mathrm{fb}$ for $m_{A^{0}}=250 \mathrm{GeV}$, where $f=$ $f_{t c}$ is the constant appearing in (2). Though the cross section is very small, it is proportional to $f^{2}$ hence a direct probe to FCNH coupling $\xi_{t c}$. At the LHC with $\sqrt{s}=14 \mathrm{TeV}$, the colliding parton momentum fractions could be much smaller so that both charm and gluon distribution functions contribute significantly. Repeating the calculation for LHC, with $m_{A^{0}}=250$ $\mathrm{GeV}$ we obtain a cross section of $37 f^{2} \mathrm{fb}$ which is 3000 times larger than that at the Tevatron, one order of magnitude larger than $h^{0} A^{0}$ associated production, and grows as $f^{2}$. We show in Fig. 2 the dependence of $\sigma\left(p p \rightarrow t(\bar{t}) A^{0}+X\right)$ on $m_{A^{0}}$ with $f$ taken to be unity.

With $A^{0} \rightarrow W$ forbidden by CP invariance, $A^{0}$ decays predominantly into $t \bar{c}$ or $\bar{t} c$ in the mass range given by (3). For example, for $m_{A^{0}}=250 \mathrm{GeV}, 90 \%$ (fraction increases with $m_{A^{0}}$ ) of $A^{0}$ decays into the above final states [7], half of which pair up with the
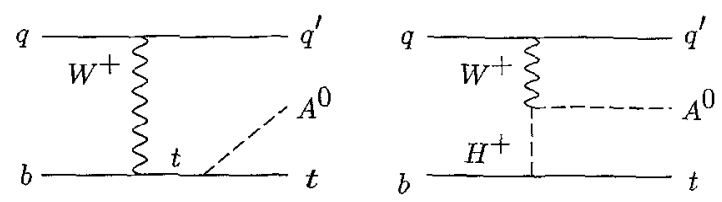

Fig. 3. Subprocess $q b \rightarrow q^{\prime} t A^{0}$.

associated top to make a like sign top pair event. The signature of such events are like sign dileptons, accompanied by two $b$-jets, large missing energy, plus one additional jet,

$c g \rightarrow t A^{0} \rightarrow \ell_{1}^{+} \ell_{2}^{+} \nu \nu+b b+\bar{c}$,

and similarly for $\bar{c} g \rightarrow \bar{t} A^{0} \rightarrow \ell_{1}^{-} \ell_{2}^{-} \bar{\nu} \bar{\nu}+\bar{b} \bar{b}+c$. With an integrated luminosity of $100 \mathrm{fb}^{-1}$ and $50 \%$ double $b$-tagging efficiency, we expect for both $\ell^{+} \ell^{+}$ and $\ell^{-} \ell^{-}$modes

$37 f^{2} \times \frac{90 \%}{2} \times \frac{4}{81} \times 50 \% \times 100=40 f^{2}$

events per year for $m_{A^{0}}=250 \mathrm{GeV}$. The event rate for other values of $m_{A^{0}}$ can be read off from Fig. 2, together with Fig. 1 of Ref. [7], where the $m_{A^{0}}$ dependence of $\mathrm{BR}\left(A^{0} \rightarrow t \bar{c}+\bar{t} c\right)$ is plotted. Over the mass range of (3), the event rate does not change significantly since $m_{A^{0}}$ affects the production cross section and $\operatorname{BR}\left(A^{0} \rightarrow t \bar{c}+\bar{t} C\right)$ in compensating ways. Although the number of events is unfortunately still on the small side, note that it is an order of magnitude larger than at a $500-600 \mathrm{GeV} e^{+} e^{-}$linear collider [7].

One might think that the same final state may also be reached by single top production followed by $A^{0}$ bremsstrahlung, as shown in Fig. 3. This is analogous to the production of a Higgs boson associated with a single top [9]. In the current context, we have

$q b \rightarrow q^{\prime} t A^{0}$.

Since the simpler parent process, the so-called singletop production $q b \rightarrow q^{\prime} t$, has a cross section around $100 \mathrm{pb}[10]$, the process (6) would appear to have a large cross section. Adding an $A^{0}$ to the final state tends to reduce the cross section by 3 orders of magnitude, but a cross section for $q b \rightarrow q^{\prime} t A^{0}$ around 100 $\mathrm{fb}$ is still quite large.

To ascertain this, we divide the total cross section into three parts, $\sigma=\sigma_{t}+\sigma_{H^{+}}+\sigma_{t H^{+}}$, where the first two terms are from each diagram alone, and the third 

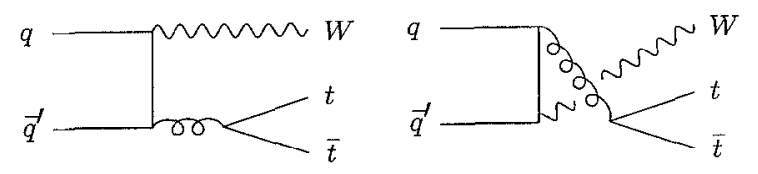

Fig. 4. Standard model $q \bar{q}^{\prime} \rightarrow W t \bar{t}$ subprocess.

is their interference. With CTEQ3L [8] parton distribution functions, we find $\sigma_{t}, \sigma_{H^{+}}$, and $\sigma_{t H^{+}}$to be 21.7, 24.4 and $-43.6 \mathrm{fb}$, respectively, for $m_{H^{+}}=m_{A^{0}}=250$ $\mathrm{GeV}$. The interference term almost cancels the diagonal terms completely and renders a total cross section of only $2.5 \mathrm{fb}$, which is much smaller than $c g \rightarrow t A^{0}$ ! The result is found to be not very sensitive to $m_{H^{+}}$ for $m_{H^{+}}>m_{A^{0}}$, and was double checked with helicity methods $[10]$. Such cancellation is pretty much a consequence of unitarity, and is rather analogous to the SM $q b \rightarrow q^{\prime} t H^{0}$ case [9].

We have now singled out $c g \rightarrow t A^{0}$ as the most promising mode to probe the FCNH coupling $\xi_{t c}$. This is a direct probe of $\xi_{t c}$, since $f$ can be determined from the cross section of $c g \rightarrow t A^{0}$. The method depends only on the mass of $A^{0}$ and the decay branching ratio for $A^{0} \rightarrow t \bar{c}(\bar{t} c)$ to givc likc-sign top quark pair events. Furthermore, because $A^{0}$ is CP-odd, the above decay branching ratio is almost model-independent for the values of $m_{A^{0}}$ condsidered. What remains to be checked are the backgrounds. We focus on like sign $W$ pair production ${ }^{1}$, which would also give rise to like sign dilepton events.

Vector boson pair production has been studied extensively [11] for the purpose of probing the electroweak symmetry breaking mechanisms. By requiring two $b$-jets and like sign dileptons in the final state, one can suppress almost all of these. Surprisingly, however, the $2 \rightarrow 3$ process $q \bar{q}^{\prime} \rightarrow W^{+}\left(W^{-}\right) t \bar{t}[12]$ of Fig. 4 turns out to be substantial. The production and decay chain

$u \bar{d} \rightarrow W^{+} t \bar{t} \rightarrow W^{+} W^{+} W^{-} b \bar{b} \rightarrow \ell_{1}^{+} \ell_{2}^{+} \nu \nu+b \bar{b}+j_{1} j_{2}$,

leads to like sign dileptons as well as a pair of $b$ - and $\bar{b}$-jets (and likewise for $d \bar{u} \rightarrow W^{-} t \bar{t} \rightarrow$ $\left.W^{-} W^{-} W^{+} b \bar{b} \rightarrow \ell_{1}^{-} \ell_{2}^{-} \bar{\nu} \bar{\nu}+b \bar{b}+j_{1} j_{2}\right)$. Unlike the

\footnotetext{
${ }^{1}$ If charge misassignment or other fake rates are high, $t \bar{f} g$ events could become a potential background. However, we take this as an experimental problem.
}

signal process of (4), there are two jets $j_{1}$ and $j_{2}$, which should have pair mass $m_{j j}$ around $M_{W}$. Convoluting with parton distribution functions, we find $\sigma\left(p p \rightarrow W^{+} t \vec{t}+X\right)=210 \mathrm{fb}$ while $\sigma(p p \rightarrow$ $\left.W^{-} t \vec{t}+X\right)=100 \mathrm{fb}$, which agrees with the results of Barger et al., Ref. [12]. The factor of two comes from the dominance of valence contributions, i.e. $u(x)=2 d(x)$ and $\bar{d}(x)=\bar{u}(x)$ for $p p$ initial state.

Assuming $100 \mathrm{fb}^{-1}$ per year at the LHC, the annual event number for process (7) is

$210 \times \frac{2}{3} \times \frac{4}{81} \times 50 \% \times 100=350$,

and half this rate for $\ell^{-} \ell^{-}+X$ events. The factor of $2 / 3$ is the $W \rightarrow j j$ branching ratio. The background of (8) appears to dominate over the signal of (5) both in $\ell^{+} \ell^{+}$and $\ell^{-} \ell^{-}$modes, though it is less severe in the latter case. Adding to the problem, we find that the $W$ boson associated with the $t \vec{t}$ pair also turns out to be produced in the central region, hence a Monte Carlo study is needed to separate signal from background. While details of such a study will be presented elsewhere, let us provide a qualitative argument on this matter. The simplest way is clearly jet counting. Two $b$-jets are already tagged, but it may be too costly to determine $b$ vs. $\breve{b}$. The signal has one additional jet while the background has two, with $m_{j j} \simeq M_{W}$. If the two background jets are both in the central region $(|\eta|<3$, where $\eta$ is pseudorapidity) and can be distinguished, the event can be excluded by naïve jet counting. If the two jets merge into one large jet $J$, the event can still be effectively removed by cutting on large $m_{J}$ around $M_{W}$. Only if either $j_{1}$ or $j_{2}$ falls outside of the detection region or coalesce accidentally with one of the $b$-jets will the event become an irreducible background. This kinematics is however unlikely because the $t \bar{t}$ syetem, which gives rise to $W \rightarrow j_{1} j_{2}$, is centrally produced as discussed before. A conservative estimate is that by jet counting alone, one should be able to reduce the background by at least $50 \%$ [13].

With simple jet counting, one has less than 90 background $\ell^{-} \ell^{-}$events, with an excess of $\sim 40 f^{2}$ coming from signal events. For $f \sim \sqrt{2}$, signal and background event rates would be comparable. In other words, if the FCNH coupling $\xi_{t c}=f \sqrt{m_{c} m_{t}} / v$ indeed exists, considerable excess could be observed in $\ell^{-} \ell^{-}$ events. For a slightly larger $f$, say $f \sim 2$, the signal is 
also comparable to the background in the $\ell^{+} \ell^{+}$mode. Defining $N\left(\ell^{ \pm} \ell^{ \pm}\right)$as the number of $\ell^{ \pm} \ell^{ \pm}$events, the signal could then manifest itself in the asymmetry parameter

$A=\frac{N\left(\ell^{+} \ell^{+}\right)-N\left(\ell^{-} \ell^{-}\right)}{N\left(\ell^{+} \ell^{+}\right)+N\left(\ell^{-} \ell^{-}\right)}$.

The background alone gives $A=\frac{1}{3}$, while the signal events lower $A$ to $\frac{1}{7}$ for $f \sim 2$.

Though interesting in itself, it should be noted that $q \bar{q}^{\prime} \rightarrow W t \bar{t}$ enters as background only because it is difficult to tag the top flavor at present (see footnote 1). To demand same-flavor $b$ tag might be too costly in terms of efficiency. The signal to background ratio would be greatly improved once $t$ vs. $\bar{t}$ can be easily distinguished experimentally. The development of such techniques should be pursued with priority at the LHC and for the longer term future, since flavor and $\mathrm{CP}$ violation are closely linked. Eventually we would like to study top flavor violation as a probe of $\mathrm{CP}$ violation in $t \vec{t}$ production and decay.

We stress that $c g \rightarrow t A^{0}$ can be viewed as a model independent probe to FCNH couplings. To produce like sign top events, only a sizable $A^{0} t c$ coupling $\xi_{t c}$ and a fairly large branching ratio for $A^{0} \rightarrow t \bar{c}+\bar{t} c$ are essential. If the first condition is satisfied, it is very probable that the second holds as well. One simply needs to argue that the branching ratio for two boson decays, $A^{0} \rightarrow W$, is suppressed. This should indeed be the case since $C P$ appears to be a good approximate symmetry, and $A^{0} W$ coupling can only be generated by loop corrections. Moving into the model independent realm, we note in passing that $u g \rightarrow t A^{0}$ could be much more prominent than discussed here, if $\xi_{u t}$ is comparable to $\xi_{c t} \sim \sqrt{2 m_{c} m_{t}} / v \sim 0.1$. In this case the large valence distribution function leads to a $t A^{0}$ cross section of order $500 \mathrm{fb}$ for $m_{A^{0}}=250 \mathrm{GeV}$, but with little gain for $\bar{t} A^{0}$. As a final remark, we note that $g g \rightarrow$ $A^{0} \rightarrow t \bar{c}$ cross section is considerably larger than those discussed here, but would be plagued by the much larger single top and $t \vec{t}$ cross sections at the LHC.
In summary, we have discussed the possibility of directly probing FCNH couplings at the LHC, via like sign top quark pair production through the $\mathrm{cg} \rightarrow$ $t A^{0} \rightarrow t t \bar{q}$ process. Possible backgrounds are identified and calculated. To better distinguish the signal from backgrounds would require a detailed Monte Carlo study. More efficient top flavor tagging ( $t$ vs. $\bar{t}$ !) methods are desirable to convincingly observe the possible production of like-sign top quark pairs.

We thank Darwin Chang, Jyh-Liong Lim and GongPing Yeh for useful discussions. The works of WSH, GLL and CYM are supported in part by National Science Council of R.O.C. under grant numbers NSC 862112-M-009-026 and NSC 86-2112-M-009-012. CPY is supported in part by NSF grant No. PHY-9507683.

\section{References}

[1] W.S. Hou, Phys. Lett. B 296 (1992) 179.

[2] L.J. Hall and S. Weinberg, Phys. Rev. D 48 (1993) R979.

[3] S.L. Glashow and S. Weinberg, Phys. Rev. D 15 (1977) 1958.

[4] T.P. Cheng and M. Sher, Phys. Rev. D 35 (1987) 3484.

[5] M. Luke and M.J. Savage, Phys. Lett. B 307 (1993) 387.

[6] D. Atwood, L. Reina and A. Soni, Phys. Rev. D 53 (1996) 1199; D 55 (1997) 3156; Phys. Rev. Lett. 75 (1995) 3800.

[7] W.S. Hou and G.L. Lin, Phys. Lett. B 379 (1996) 261.

[8] H.L. I ai et al., Phys. Rev. D 51 (1995) 4763.

[9] W.J. Stirling and D.J. Summers, Phys. Lett. B 283 (1992) 411 ;

A. Ballestrero and E. Maina, ibid. B 299 (1993) 312;

G. Bordes and B. Van Eijk, ibid. B 299 (1993) 315;

A. Ballestrero et al., ibid. B 320 (1994) 305.

[10] D. Carlson and C.-P. Yuan, Pliys. Lett. B 306 (1993) 386; C.-P. Yuan, talk given at the Workshop on Physics of the Top Quark, Iowa State University, Ames, Iowa, May 25-26, 1995;

D. Carlson, Phi.D. thesis, Michigan State University, 1995.

[11] See for example, J, Bagger et al., Phys. Rev. D 49 (1994) 1246;

D 52 (1995) 3878; and references therein.

[12] Z. Kunszt, Nucl. Phys. B 247 (1984) 339;

V. Barger et al., Phys. Rev. D 42 (1990) 3052.

[13] G.P. Yeh, private communications. 